\title{
A genome-wide association study reveals novel genomic regions and positional candidate genes for fat deposition in broiler chickens
}

Gabriel Costa Monteiro Moreira ${ }^{1}$, Clarissa Boschiero ${ }^{1}$, Aline Silva Mello Cesar ${ }^{1}$, James M. Reecy², Thaís Fernanda Godoy ${ }^{1}$, Priscila Anchieta Trevisoli ${ }^{1}$, Maurício E. Cantão ${ }^{3}$, Mônica Corrêa Ledur ${ }^{3}$, Adriana Mércia Guaratini Ibelli ${ }^{3}$, Jane de Oliveira Peixoto ${ }^{3}$, Ana Silvia Alves Meira Tavares Moura ${ }^{4}$, Dorian Garrick ${ }^{5}$ and Luiz Lehmann Coutinho ${ }^{1 *}$

\begin{abstract}
Background: Excess fat content in chickens has a negative impact on poultry production. The discovery of QTL associated with fat deposition in the carcass allows the identification of positional candidate genes (PCGs) that might regulate fat deposition and be useful for selection against excess fat content in chicken's carcass. This study aimed to estimate genomic heritability coefficients and to identify QTLs and PCGs for abdominal fat (ABF) and skin (SKIN) traits in a broiler chicken population, originated from the White Plymouth Rock and White Cornish breeds.

Results: ABF and SKIN are moderately heritable traits in our broiler population with estimates ranging from 0.23 to 0.33. Using a high density SNP panel (355,027 informative SNPs), we detected nine unique QTLs that were associated with these fat traits. Among these, four QTL were novel, while five have been previously reported in the literature. Thirteen PCGs were identified that might regulate fat deposition in these QTL regions: JDP2, PLCG1, HNF4A, FITM2, ADIPOR1, PTPN11, MVK, APOA1, APOA4, APOA5, ENSGALG00000000477, ENSGALG00000000483, and ENSGALG00000005043. We used sequence information from founder animals to detect 4843 SNPs in the 13 PCGs. Among those, two were classified as potentially deleterious and two as high impact SNPs.

Conclusions: This study generated novel results that can contribute to a better understanding of fat deposition in chickens. The use of high density array of SNPs increases genome coverage and improves QTL resolution than would have been achieved with low density. The identified PCGs were involved in many biological processes that regulate lipid storage. The SNPs identified in the PCGs, especially those predicted as potentially deleterious and high impact, may affect fat deposition. Validation should be undertaken before using these SNPs for selection against carcass fat accumulation and to improve feed efficiency in broiler chicken production.
\end{abstract}

Keywords: Genomic heritability, QTL, Abdominal fat, Skin weight, Fatness

\footnotetext{
* Correspondence: Ilcoutinho@usp.br

'Department of Animal Science, University of São Paulo (USP) / Luiz de

Queiroz College of Agriculture (ESALQ), Piracicaba, São Paulo 13418-900,

Brazil

Full list of author information is available at the end of the article
}

(c) The Author(s). 2018 Open Access This article is distributed under the terms of the Creative Commons Attribution 4.0 International License (http://creativecommons.org/licenses/by/4.0/), which permits unrestricted use, distribution, and reproduction in any medium, provided you give appropriate credit to the original author(s) and the source, provide a link to the Creative Commons license, and indicate if changes were made. The Creative Commons Public Domain Dedication waiver (http://creativecommons.org/publicdomain/zero/1.0/) applies to the data made available in this article, unless otherwise stated. 


\section{Background}

The chicken was the first domesticated animal species that was whole-genome sequenced and it has emerged as an excellent model for genomic studies in agriculture, developmental biology, fatness and leanness [1]. The main fat deposits in chicken are located in the skin (including subcutaneous fat) and within the abdominal cavity (abdominal plate) [2-4]. Excess fat deposition in broiler chickens is a negative factor for the poultry industry because it decreases feed efficiency and reduces the nutritional value of carcass parts and, consequently, their commercial value [5-7].

Broiler chicken lines have been selected for rapid growth, and carcass yield [7, 8]. Rapid growth results in increased fat deposition within the carcass [8] and commercial chickens exhibit higher fat deposition compared with unselected chickens [7]. The selection of chickens for rapid growth and reduced carcass fat deposition is challenging because these two traits have a positive genetic correlation [8].

Some studies have been conducted to map genomic quantitative trait loci (QTLs) associated with variation in abdominal fat [5, 9-13], and skin traits [9, 14]. However, most previously published QTLs were mapped using low density of markers (ranging from 102 to 410 markers), and the detected intervals spanned tens of centimorgans (cM) [15].

Previous genome-wide association studies (GWAS) have been performed for abdominal fat weight and fat percentage in an F2 chicken population's using a $60 \mathrm{~K}$ SNP chip (Illumina) $[16,17]$ and in a local population of a local Chinese breed using approximately 90,000 SNPs [18]. To the best of our knowledge, no GWAS was reported for fatness traits in a meat-type population using the high-density SNP chip (600 K) from Affymetrix [19].

Fat deposition is an economically-relevant trait in fast-growing chickens, and knowledge about the genetic regulation of this trait is essential for breeding programs. Based on this fact, the main goal of this study was to perform GWAS analysis using a high-density SNP panel $(600 \mathrm{~K})$ to identify QTLs and positional candidate genes (PCGs) and possibly candidate mutations for fat deposition in broiler chickens.

\section{Methods}

All experimental protocols related to animal experimentation in this study were performed in agreement with resolution number $011 / 2010$ approved by the Embrapa Swine and Poultry Ethics Committee on Animal Utilization (CEUA) in Concordia, Santa Catarina State South of Brazil, in agreement with the rules of National Council of Animal Experimentation Control (CONCEA) to ensure compliance with international guidelines for animal welfare.

\section{Chicken population}

This study was conducted with a paternal broiler line (TT) belonging to the Chicken Breeding Program of EMBRAPA Swine and Poultry National Research Center, in Concordia, Santa Catarina State - South of Brazil. This line, originating from the White Plymouth Rock and White Cornish breeds, has been under multiple trait selection since 1992 mainly for body weight, feed conversion, carcass and cuts yield, viability, fertility, hatchability and reduced abdominal fat [13, 20-23]. The TT Reference Population evaluated in this study was developed in 2008 and consisted of 1430 chickens (652 males and 778 females) generated in five hatches from 20 males and 92 females (1:5). Previous genomic studies have been performed in this population, and more details can be found in [20-23].

\section{Phenotype measurement}

After $6 \mathrm{~h}$ of fasting, the chickens at 42 days of age were weighted (BW42) and then euthanized by cervical dislocation. In this step, a blood sample from each chicken was immediately collected for subsequent DNA extraction then, the carcass was cooled. After $4 \mathrm{~h}$ of cooling, the weights of the carcass, skin covering each carcass part (thigh, drumstick, and breast) and abdominal fat (abdominal fat pad) were measured. The percentage of each trait was calculated dividing the weigh by BW42 and multiplying by 100 . Total skin weight and percentage were used as indicators of subcutaneous fat, as discussed by Zerehdaran et al. [3]. More details about the rearing conditions and phenotypes measurements are available in Fornari et al. [22].

\section{DNA extraction, genotyping and quality control}

Genomic DNA from 1430 blood samples were extracted using the PureLink ${ }^{\oplus}$ Genomic DNA (Invitrogen, Carlsbad, CA, USA) kit and were quantified using Qubit ${ }^{\oplus} 2.0$ Fluorometer (Thermo Fisher Scientific, Waltham, MA, USA). After extraction, DNA integrity was evaluated on agarose gel (1\%) and diluted to $10 \mathrm{ng} / \mu \mathrm{L}$. Diluted genomic DNA was prepared following recommended Affymetrix protocols in order to perform the genotyping analysis using $600 \mathrm{~K}$ Affymetrix Axiom Genotyping Array (Affymetrix, Inc. Santa Clara, CA, USA). This genotyping array comprises segregating SNPs for different chicken populations, including four commercial broilers (broiler chicken lines), as detailed by Kranis et al. [19].

Initially, Axiom $^{\mathrm{T}}{ }^{\mathrm{M}}$ Analysis Suite (Affymetrix ${ }^{\circledR}$ ) software was used to filter genotypes based on the DishQC parameter, after which PLINK v.1.9 [24] software was used to perform quality control analysis and for genotype calling. Only samples that exhibited DishQC $\geq 0.82$ and call rate $\geq 90 \%$ were kept for further analysis. Considering these retained samples, in 
order to select markers with high quality, and to avoid potential genotyping errors or even DNA contamination, further edits were undertaken based on literature recommendations [25], to remove single nucleotide polymorphisms with a call rate $\leq 98 \%$, minor allele frequency (MAF) $\leq 2 \%$ or significant deviations from HWE ( $p$-value $<0.000001$ ). Single nucleotide polymorphisms located in the sex chromosomes, and those SNPs not mapped in the chicken assembly (Gallus_gallus-5.0, NCBI) were excluded from the analysis. Only the SNPs annotated to autosomal chromosomes from GGA1 to GGA28 were used in statistical analyses. After all the filtering steps, the few remained missing genotypes were replaced by the average of covariate values at that particular locus, as described by Cesar et al. [26].

From a total of 1430 genotyped chickens, 22 samples were removed from the analysis after applying the DishQC criteria, and a filter on sample call rate $\geq 90 \%$ loci. From the total of 580,961 SNPs available on the SNP array, 355,027 informative polymorphic SNPs on the autosomal chromosomes (GGA1-28) were kept after filtering. The average density of SNPs was 520 SNPs/ $\mathrm{Mbp}$, with the lowest chromosome-wise density observed on GGA2 (268 SNPs/Mbp), and the highest chromosome-wise density on GGA21 (898 SNPs/Mbp) (Additional file 1).

\section{Descriptive statistics and heritability}

The mean and the standard deviation of each phenotype were calculated using $\mathrm{R}$ scripts. The estimation of variance components (genetic variance, residual variance, and total variance) was performed using a Bayes C model in GenSel software [27] using the samples and SNPs that remained after genotyping and filtering. The resultant posterior means of the variance components were used as priors in subsequent Bayes B models to estimate genomic heritability for each trait.

\section{Genome wide association analysis}

The SNPs that passed the quality control filters were used in the GWAS analysis using genomic prediction methodology with a Bayesian approach in GenSel software [27]. In the first step, a Bayes $C$ model was used to estimate the genetic and residual variances for each trait and these values were then used as priors to run a Bayes B model as performed by Cesar et al. [26]. The Bayes B models sample the effects of SNPs assuming some fraction of the effects are zero and with unequal variance of each effect [28]. The mathematical model was:

$$
\boldsymbol{y}=\boldsymbol{X} \boldsymbol{b}+\sum_{j=1}^{k} \boldsymbol{a}_{j} \beta_{j} \delta_{j}+\boldsymbol{e}
$$

In this model, $y$ represents the vector of phenotypic values; $\boldsymbol{X}$ is the incidence matrix for fixed effects; $\boldsymbol{b}$ is the vector of fixed effects; $K$ is the number of SNPs; $a_{j}$ is the column vector representing the SNP as a covariate in locus ${ }_{j}$ coded with the number of B alleles; $\beta j$ is the random substitution effect for locus $j$ assumed to be normally distributed $N\left(0, \sigma_{\beta i}^{2}\right)$ when $\delta j=1$ but $\beta j=0$ when $\delta j=0$, with $\delta j$ being a random variable $0 / 1$ indicating the absence (with probability $\pi$ ) or presence (with probability $1-\pi$ ) of the locus $j$ in the model, and $\boldsymbol{e}$ is the residual associated with the analysis. Sex and hatch were included as fixed effects in the model and BW42 (slaughter age) as a fixed covariate for ABF and SKINW.

We assumed $\pi=0.9970$ in the BayesB models and obtained 41,000 Markov chain Monte Carlo (MCMC) samples with the first 1000 samples being discarded. A map file was used to position the markers into 947 non-overlapping $1 \mathrm{Mb}$ windows. The windows that had the marker with higher model frequency in the MCMC interactions had their effect predicted as mentioned by Van Goor et al. [29]. Each window is expected to explain $0.1054 \%$ of the genetic variance $(100 \% / 947)$ based on an infinitesimal model $[30,31]$, and windows that explained five times more than the expected $(0.53 \%)$ were considered significant. Thus, we selected only significant windows to characterize and identify PCGs.

\section{Overlap with known QTLs}

The overlap of our genomic windows with previously mapped QTLs for fat-related traits in chickens was determined using the information available at Chicken QTLdb - release 33, accessed in September, 2017 [32]. We used the available BED file with the QTL coordinates according to the last chicken genome assembly (Gallus_gallus-5.0, NCBI) to check the overlaps using in-house $\mathrm{R}$ scripts. The genomic windows that did not overlap with previously annotated QTLs for fat traits were considered to be novel discoveries. All the previously mapped QTLs were reported by QTL ID numbers, available at Chicken QTLdb [32].

\section{Identification of positional candidate genes}

A list of annotated genes within each QTL (genomic window) and their respective GO terms and biological processes were obtained using Ensembl BioMart tool $[33,34]$. Genes that had GO terms and a biological process related to fat deposition were initially selected. Next, two different databases (NCBI, OMIM) were searched to identify existing literature to support/refute the positional candidate genes (PCG) identified. 
Enrichment analysis of gene list was performed with the Functional Annotation Tool (FAT) in Database for Annotation, Visualization and Integrated Discovery software (DAVID bioinformatics resources v.6.8, [35, 36]) to identify enriched clusters of genes. To select a gene cluster as enriched, we considered an enrichment score > 1.00 and within the cluster, GO terms for biological process (BP) with a raw $p$-value $<0.05$ and p-value adjusted for multiple testing by Benjamini \& Hochberg [37] method $<0.1$.

\section{Candidate genes screening for SNPs from sequencing data}

To refine our list of candidate genes, we screened our PCGs for genetic variants using a dataset of SNPs identified in the parental generation from our genotyped population, which were generated by next generation sequencing of 14 parental males (from 112) with approximately $13 \mathrm{X}$ of coverage performed by HiScanSQ (Illumina) with a read length of 101 bp. Further details about library preparation and sequencing are available in Moreira et al. [38] and Godoy et al. [39].

SNP calling was performed using the most recent chicken genome assembly Gallus-gallus-5.0 (UCSC) with SAMtools software v.1.2 [40], with mapping and base qualities (Phred score) $\geq 20$. The filtering criteria and further details about SNP calling are available in Boschiero et al. [41]. After variant filtering, the SNP dataset was annotated using Variant Effect Predictor (VEP) tool version 86 [42]. SNP density were determined considering all the unique SNPs annotated (including $5 \mathrm{~Kb}$ up and downstream) in each PCG and its gene length.

Variants located in coding regions can lead to phenotypic changes [38, 39, 43]. To predict whether SNPs that caused changes in amino acids were tolerant or not (may affect the function of the gene product), we calculated the SIFT (sorting intolerant from tolerant) score. This score is an assessment of the level of conservation in homologous protein sequences using the SIFT algorithm [44] implemented by the VEP tool version 86 [42]. SIFT scores were calculated for all the non-synonymous and stop codon (gained/lost) variants located in the PCGs.

High impact SNPs were also evaluated in the candidate genes. The VEP tool [42] provides an estimation of the putative impact of the variant classified as high impact, i.e. annotating all the mutations annotated as transcript ablation, splice acceptor, splice donor, stop gained, frameshift, stop loss, start lost and transcript amplification, mutations that may cause protein truncation, loss of function or trigger nonsense mediated decay [43].

\section{Results}

\section{Descriptive statistics and genomic heritability}

The number of animals, averages and standard errors, variance components and estimated genomic heritability from the Bayes B model are given in Table 1 for ABF, ABFP, SKINW and SKINP. We estimated genomic heritability values to be moderate for all traits evaluated; $A B F$ traits exhibited higher genomic heritability compared to SKIN traits.

\section{Genome wide association analysis (GWAS)}

A list with all the SNP windows analyzed including the proportion of the genetic variance explained by each one (even those with effects close to zero) is provided in Additional file 2. The QTLs (significant genomic windows) associated with fat deposition are described in Table 2. Nine unique significant $1 \mathrm{Mb}$ windows (with different unique positions) were identified on GGA 5, 9, $10,13,15,20,24,26$, and 27 . The posterior probability of association (PPA), as described by Onteru et al. [31], ranged from 0.82 to 0.95 for each region, and the proportion of genetic variance explained by the window ranged from 0.54 to 1.49 .

The Manhattan plot of the posterior means of the proportion of genetic variance explained by each SNP window across the 28 autosomal chromosomes for ABF are presented in Fig. 1. The Manhattan plots for ABFP, SKINW and SKINP are in Additional files 3, 4, and 5, respectively.

In order to support our findings, we checked the effect of the markers within the associated genomic windows. Manhattan plots of the SNP effect distribution within each significant SNP window for ABF are colored by chromosome and presented in Fig. 2. The Manhattan plots for ABFP, SKINW and SKINP are in Additional files 6, 7, and 8, respectively.

\section{Overlap with previously reported QTLs}

Twenty-seven previously published QTLs for fat traits overlapped with five of the QTLs identified in our study. The QTL located on GGA5 at $38 \mathrm{Mb}$, associated with $\mathrm{ABF}$ and ABFP overlapped with three known QTLs: two QTLs associated with for ABF (QTL \#3321, [45]; QTL \#9432, [46]) and one associated with ABFP (QTL \#9433, [46]).

The QTL, which was located on GGA15 at $6 \mathrm{Mb}$ and associated with SKINW and SKINP, overlapped with 11 QTLs previously associated with fat traits: four were associated with for ABF (QTL \#2337, [5]; QTL \#9451, [46]; QTL \#2347, QTL \#12631, [9]), three were associated with ABFP (QTL \#2339, QTL \#2340, [5]; QTL \#9450, [46]), one associated with fat distribution (total weight of skin fat analyzed with ABF as covariate) (QTL \#12645, [9]), one QTL associated with subcutaneous 
Table 1 Descriptive statistics, variance components and genomic heritability for body weight at 42 days of age, abdominal fat and skin weights and percentages in the $\Pi$ Reference Population

\begin{tabular}{lllllll}
\hline Trait & $\mathrm{N}$ & Average $\pm \mathrm{SD}^{\mathrm{a}}$ & Genetic variance & Residual Variance & Total variance & Genomic heritability $^{\mathrm{b}}$ \\
\hline BW42 & 1311 & $2220.30 \pm 258.86$ & $12,378.000$ & $25,423.100$ & $37,801.100$ & 0.33 \\
ABF & 1287 & $47.10 \pm 14.03$ & 46.599 & 96.079 & 142.677 & 0.33 \\
ABFP & 1287 & $2.13 \pm 0.62$ & 0.094 & 0.205 & 0.299 & 0.31 \\
SKINW & 1303 & $94.55 \pm 16.12$ & 29.936 & 96.443 & 126.379 & 0.23 \\
SKINP & 1303 & $4.25 \pm 0.56$ & 0.063 & 0.203 & 0.266 & 0.23 \\
\hline
\end{tabular}

BW42: body weight at 42 days of age in grams; $A B F$ abdominal fat weight in grams, ABFP abdominal fat percentage, SKINW skin weight in grams, SKINP skin percentage

a Standard deviation of the mean

${ }^{\mathrm{b}}$ Genomic heritability estimated by Bayes B model

neck fat weight (QTL \#17331, [2]), one QTL associated with total white fat weight (QTL \#17337, [2]), and one associated with visceral fat weight (QTL \#17323, [2]).

The QTL located on GGA24 at $5 \mathrm{Mb}$ that was associated with SKINW, overlapped with one QTL previously reported to be associated with ABF (QTL \#9405, [47]). While, the QTL identified on GGA26 at $1 \mathrm{Mb}$ that was associated with $\mathrm{ABF}$ and $\mathrm{ABFP}$, overlapped with two QTLs: one associated with visceral fat weight (QTL \#17324, [2]), and one associated with total white fat weight (QTL \#17338, [2]).

The QTL identified on GGA27 at $3 \mathrm{Mb}$ that was associated with SKINW and SKINP, overlapped with 10 QTLs previously reported to be associated with fat traits: three associated with ABF (QTL \#66072, [48]; QTL \#11817, QTL \#11809, [49]), three associated with ABFP
(QTL \#11820, [49]; QTL \#3354, [50]; QTL \#11934, [51]), two associated with carcass fat content (QTL \#17135, QTL \#17126, [13]), one associated with carcass fat content on a dry matter basis (QTL \#17125, [13]), and one associated with intramuscular fat (QTL \#3360, [50]).

No previously reported QTL overlapped with the QTL identified on GGA9 at $4 \mathrm{Mb}$, GGA10 at $7 \mathrm{Mb}$, GGA13 at $3 \mathrm{Mb}$, and GGA20 at $5 \mathrm{Mb}$.

\section{Positional candidate genes}

We identified 419 genes in the nine QTL genomic windows (Additional file 9). Further analysis against gene ontology terms and the existing literature revealed 13 candidate genes for fat deposition (Table 3).

Table 2 Characterization of $1 \mathrm{Mb}$ significant genomic windows for abdominal fat and skin traits in the TT Reference Population

\begin{tabular}{|c|c|c|c|c|c|c|}
\hline Trait & GGA $(M b)^{a}$ & $\begin{array}{l}\text { SNP window } \\
\text { (first - last position) }^{\mathrm{a}}\end{array}$ & $\begin{array}{l}\text { Number of } \\
\text { SNP/ window }\end{array}$ & $\begin{array}{l}\text { Number of } \\
\text { genes/ window }\end{array}$ & $\begin{array}{l}\text { Proportion of genetic variance } \\
\text { explained by the SNP window }\end{array}$ & $\mathrm{PPA}^{\mathrm{C}}$ \\
\hline \multirow[t]{5}{*}{$\overline{A B F}$} & $5(38)$ & $38,000,437-38,996,916$ & 396 & 31 & 0.92 & 0.84 \\
\hline & $10(7)$ & $7,000,336-7,998,549$ & 592 & 21 & 0.58 & 0.93 \\
\hline & $13(3)$ & $3,002,617-3,998,616$ & 460 & 16 & 1.45 & 0.88 \\
\hline & $20(5)$ & $5,000,651-5,999,452$ & 492 & 53 & 0.94 & 0.88 \\
\hline & $26(1)$ & $1,002,598-1,999,851$ & 662 & 74 & 1.06 & 0.95 \\
\hline \multirow[t]{4}{*}{ ABFP } & $5(38)$ & $38,000,437-38,996,916$ & 396 & 31 & 0.64 & 0.82 \\
\hline & $10(7)$ & $7,000,336-7,998,549$ & 592 & 21 & 0.61 & 0.90 \\
\hline & $13(3)$ & $3,002,617-3,998,616$ & 460 & 16 & 1.49 & 0.89 \\
\hline & $26(1)$ & $1,002,598-1,999,851$ & 662 & 74 & 0.54 & 0.92 \\
\hline \multirow[t]{3}{*}{ SKINW } & $15(6)$ & $6,000,311-6,999,944$ & 544 & 62 & 0.73 & 0.89 \\
\hline & $24(5)$ & $5,000,105-5,999,010$ & 778 & 60 & 0.56 & 0.91 \\
\hline & $27(3)$ & $3,000,222-3,997,124$ & 933 & 52 & 0.60 & 0.94 \\
\hline \multirow[t]{3}{*}{ SKINP } & $9(4)$ & $4,000,836-4,999,336$ & 482 & 50 & 0.73 & 0.83 \\
\hline & $15(6)$ & $6,000,311-6,999,944$ & 544 & 62 & 0.71 & 0.91 \\
\hline & $27(3)$ & $3,000,222-3,997,124$ & 933 & 52 & 0.57 & 0.95 \\
\hline
\end{tabular}

$A B F$ bdominal fat weight, $A B F P$ abdominal fat percentage, SKINW skin weight, SKINP skin percentage

${ }^{\mathrm{a}}$ Map position based on Gallus_gallus-5.0 assembly (NCBI)

${ }^{b}$ Number of genes annotated within the genomic window based on Ensembl Genes 90 Database

'Posterior probability of association (PPA) as described by Onteru et al. [31] 

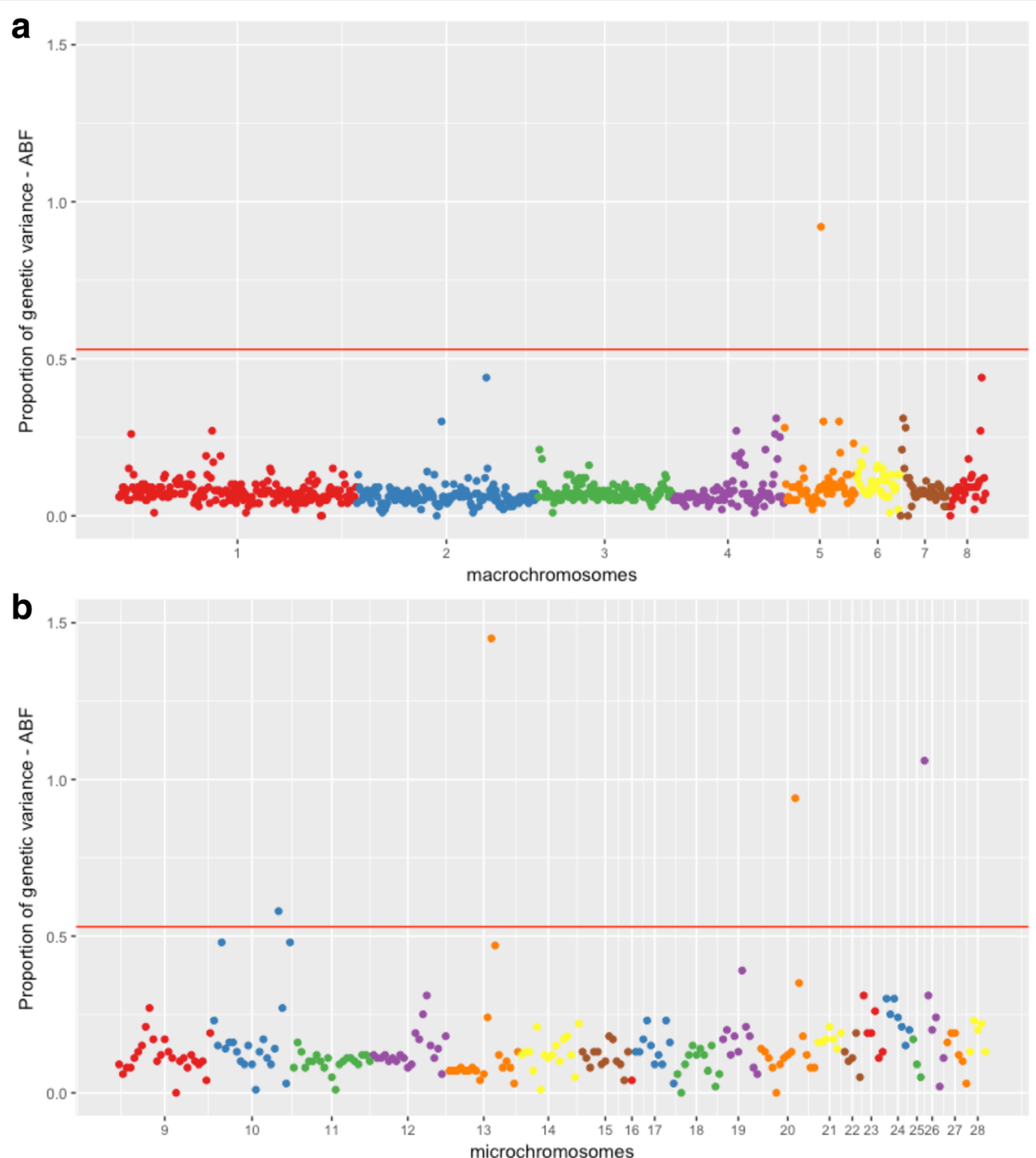

Fig. 1 Manhattan plot of the posterior means of the proportion of genetic variance explained by each 1-Mb SNP window across the 28 autosomal chromosomes for abdominal fat weight (ABF): (a) genomic windows located on macrochromosomes, and (b) windows located on microchromosomes. The $X$-axis represents the chromosomes, and $Y$-axis shows the proportion of genetic variance explained by each window from Bayes B analysis. Red lines indicate the threshold to deem significant SNP windows

Additionally, all 419 genes located within the detected QTLs were used to perform enrichment analysis. One cluster was enriched (enrichment score of 2.62) and within this cluster, four GO terms were enriched: regulation of intestinal cholesterol absorption; high-density lipoprotein particle assembly, lipoprotein metabolic process and positive regulation of fatty acid biosynthetic process (raw $p$-value $<0.05$ and $\mathrm{p}$-value adjusted for multiple testing by Benjamini \& Hochberg [37] method $<0.1)$. These GO terms were annotated for the same genes: $A P O A 1, A P O A 4$, and $A P O A 5$.

\section{SNPs in candidate genes}

A previous study has been performed using sequencing data to identify and characterize genome-wide SNPs, INDELs, putative regions under selection, and also to find putative pathways under selection in two Brazilian chicken lines [41], but neither was based on the TT broiler reference population.

We used a dataset of high quality SNPs from sequencing data identified in 14 parental chickens from TT Reference Population in order to screen for SNPs potentially affecting gene expression and/or function and identified 3639 SNPs located within the 13 PCG. SNP density (SNPs/kb) within each PCG and the functional annotation of the SNPs are presented in Fig. 3. The PCG that had the greatest density of SNPs was FITM2 (84 SNPs/kb).

Single nucleotide polymorphisms were evaluated for potentially deleterious and high impact mutation annotation, which may potentially affect gene expression and/ or function. Two high impact variants were identified: 


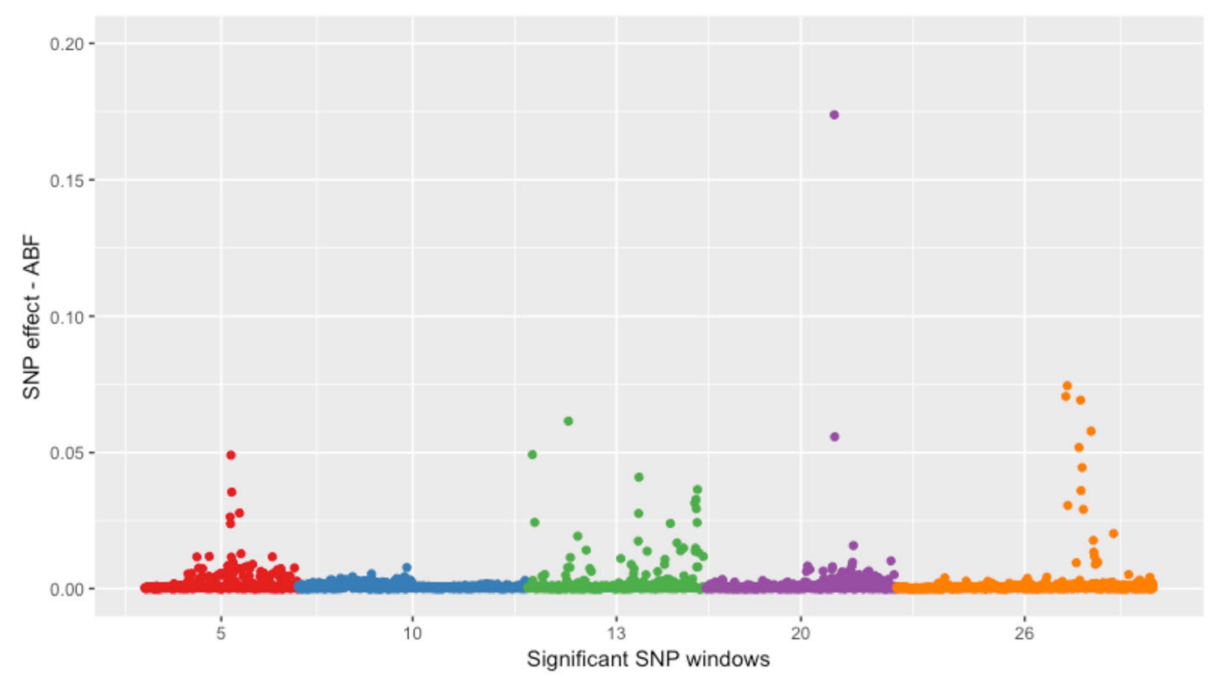

Fig. 2 Manhattan plot of the SNP effect distribution within each significant window for abdominal fat weight (ABF). The X-axis represents the significant SNP window represented by the number of the respective chromosome and Y-axis shows the SNP effect from Bayes B analysis. Their respective start and end positions are: GGA5 (38,000,437-38,996,916 bp); GGA10 (7,000,336-7,998,549 bp); GGA13 (3,002,617-3,998,616 bp); GGA20 (5,000,651-5,999,452 bp); GGA26 (1,002,598-1,999,851 bp)

one located within FITM2 and another located in ENSGALG00000000483 gene. In addition, two potentially deleterious variants were identified: one located in PLCG1 and another in ENSGALG00000000477 gene (Table 4). The non-synonymous SNP located in the PLCG1 gene is novel.

\section{Discussion}

\section{Genomic heritability}

Genomic heritability estimates for abdominal fat and skin traits in a broiler chicken population characterized by close relatives (full and half-sibs) were obtained using genotypes from a high-density SNP panel. Close relatives may have long chromosome segments in common, thereby sharing alleles and QTLs in the same pattern, which may lead to less bias and consequently, higher prediction accuracy for genomic heritability [52].

Heritabilities estimates for ABF and ABFP have been reported to be 0.62 and higher, while the heritability of skin traits is between 0.24 and 0.28 [3,53]. The number of generations of artificial selection and/or the genetic background may differ for each chicken population thus, different heritability estimates may be observed. The TT broiler line, used to obtain the TT Reference population, has been under multi-trait selection with emphasis on body weight. This trait has a positive genetic correlation with abdominal fat and feed conversion [54]. Therefore, artificial selection may also affect the genetic variance and may reduce heritability over the generations [55]. Comparisons between heritabilities reported in the literature should be interpreted with caution.
Using the same population reported here, Fornari et al. [22] observed similar pedigree-based heritability estimates to those we obtained using genomic information, namely for abdominal fat weight $(0.33)$ and two skin related traits: drumstick skin weight $(0.17)$ and thigh skin weight (0.28). The existence of moderate genomic heritabilities for the analyzed phenotypes indicates that a reasonable proportion of the total variance for these traits can be explained by a set of markers [52]. Therefore, selection against fat deposition in this population may be successful.

\section{GWAS, QTL discovery and resolution}

Bayesian approaches are commonly used in genomic prediction and selection studies [56, 57] as well as for GWAS [29, 30, 58, 59] in chickens. The main advantage of this approach is that genotypes are simultaneously fitted in the model, accounting for population structure, and the use of high-density markers does not reduce the power to detect association [60]. Thus, we decided to use genomic prediction methodology to perform GWAS.

Five out of the nine QTLs detected were previously identified in different populations, corroborating our results, and indicating that these QTLs probably originated from the founder lines used to generate the broiler TT line used in this study.

The novel QTLs identified could be false positives, exclusive to our population, or a consequence of the number of animals and the higher SNP density compared to other QTL mapping studies for abdominal fat and skin traits in chickens [5, 8-14]. The PPA (ranging from 0.83 to 0.93 ) and the proportion of the genetic variance 
Table 3 List of candidate genes within the genomic windows associated with abdominal fat and skin traits that exhibited GO terms related to lipid metabolic processes in the $\Pi$ Reference Population

\begin{tabular}{|c|c|c|c|c|}
\hline GGA (location, Mb) & Trait associated & Gene Name & Ensembl Gene ID & GO Term (GO Accession) ${ }^{a}$ \\
\hline $5(38)$ & $A B F, A B F P$ & JDP2 & ENSGALG00000010322 & negative regulation of fat cell differentiation (GO:0045599) \\
\hline \multirow[t]{6}{*}{$15(6)$} & SKINW, SKINP & PTPN11 & ENSGALG000000004821 & lipid metabolic process (GO:0006629) \\
\hline & & & & triglyceride metabolic process (GO:0006641) \\
\hline & & MVK & ENSGALG00000013848 & lipid metabolic process (GO:0006629) \\
\hline & & & & cholesterol metabolic process (GO:0008203) \\
\hline & & Novel gene & ENSGALG000000005043 & fatty acid biosynthetic process (GO:0006633) \\
\hline & & & & acetyl-CoA carboxylase activity (GO:0003989) \\
\hline \multirow[t]{8}{*}{$20(5)$} & $\mathrm{ABF}$ & FITM2 & ENSGALG000000026285 & lipid storage (GO:0019915) \\
\hline & & & & lipid particle organization (GO:0034389) \\
\hline & & PLCG1 & ENSGALG000000003750 & lipid metabolic process (GO:0006629) \\
\hline & & & & lipid catabolic process (GO:0016042) \\
\hline & & HNF4A & ENSGALG000000004285 & lipid metabolic process (GO:0006629) \\
\hline & & & & regulation of lipid metabolic process (GO:0019216) \\
\hline & & & & lipid homeostasis (GO:0055088) \\
\hline & & & & fatty acid binding (GO:0005504) \\
\hline \multirow[t]{13}{*}{$24(5)$} & SKINW & APOA4 & ENSGALG00000007109 & lipid homeostasis (GO:0055088) \\
\hline & & & & multicellular organismal lipid catabolic process (GO:0044240) \\
\hline & & & & positive regulation of triglyceride catabolic process (GO:0010898) \\
\hline & & & & cholesterol homeostasis (GO:0042632) \\
\hline & & & & cholesterol metabolic process (GO:0008203) \\
\hline & & & & positive regulation of fatty acid biosynthetic process (GO:0045723) \\
\hline & & APOA5 & ENSGALG00000014368 & triglyceride homeostasis (GO:0070328) \\
\hline & & & & positive regulation of lipid catabolic process (GO:0050996) \\
\hline & & & & positive regulation of fatty acid biosynthetic process (GO:0045723) \\
\hline & & APOA1 & ENSGALG00000007114 & lipid transport (GO:0006869) \\
\hline & & & & lipid metabolic process (GO:0006629) \\
\hline & & & & lipid storage (GO:0019915) \\
\hline & & & & cholesterol homeostasis (GO:0042632) \\
\hline \multirow[t]{11}{*}{$26(1)$} & $A B F, A B F P$ & Novel gene & ENSGALG00000000477 & lipid metabolic process (GO:0006629) \\
\hline & & & & lipid catabolic process (GO:0016042) \\
\hline & & & & lipid particle (GO:0005811) \\
\hline & & & & lipid homeostasis (GO:0055088) \\
\hline & & Novel gene & ENSGALG00000000483 & lipid catabolic process (GO:0016042) \\
\hline & & & & lipid particle (GO:0005811) \\
\hline & & & & lipid homeostasis (GO:0055088) \\
\hline & & & & triglyceride lipase activity (GO:0004806) \\
\hline & & & & triglyceride catabolic process (GO:0019433) \\
\hline & & ADIPOR1 & ENSGALG000000000094 & regulation of lipid metabolic process (GO:0019216) \\
\hline & & & & fatty acid oxidation (GO:0019395) \\
\hline
\end{tabular}

${ }^{a}$ All GO terms were obtained from BioMart (Ensembl Genes 90 Database)

explained by the novel QTLs (ranging from 0.58 to 1.49 ) suggest that these novel QTL are not false positives. Besides that, the Manhattan plots of the SNP effects within the
QTLs detected showed few peaks indicating that some markers exhibit higher effects within the QTL (see the direction of the SNP effects; Fig. 2, Additional files 6, 7, and 8) 


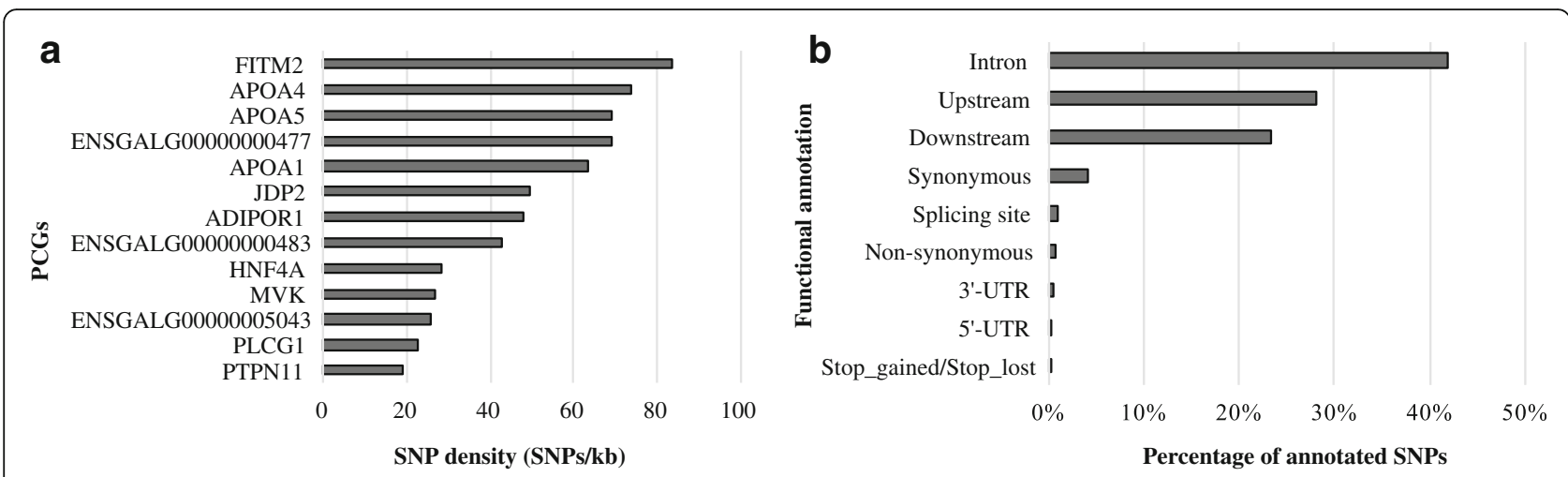

Fig. 3 a Plot of SNP density (SNPs/kb) for each PCG. b Plot with the percentage of functional annotation of SNPs identified in our 13 PCGs

providing helpful information for further studies aiming to fine-map these QTLs.

In general, we observed small effects for the markers fitted simultaneously within the QTLs detected. This could be due to lack of power to capture the genetic variability in our population or, due to artificial selection. As mentioned before, the artificial selection can lead to fixation of specific loci $[55,61]$ and SNPs with higher effect on fatness may have been fixed over the generations.

In contrast to the QTL mapped on GGA5 at $38 \mathrm{Mb}$, previously reported QTLs for the same trait were larger than $1 \mathrm{Mb}[45,46]$. The use of a higher density of markers $(600 \mathrm{~K})$ may help to explain the improved resolution observed in QTL mapping.

The QTL mapped on GGA27 at $3 \mathrm{Mb}$ overlapped with known QTLs for fatness related traits, mapped in a Brazilian F2 population established by crossing a broiler male line (TT) and a layer line (CC), and these known QTLs are segregating in different families from the Brazilian F2 population $[13,49]$. Furthermore, the broiler male line (TT) used in the crossing to establish this population, is the same line used to obtain the TT Reference Population [13] thus, we should expect this QTL segregating in our broiler population, corroborating our findings.

We identified only a few QTLs associated with fat traits in this population. For quantitative traits, a greater number of alleles are expected to present a small effect [62], and the number of samples used in this study may not have been sufficiently large enough to identify these small effect QTLs. Despite this, novel QTLs for fat traits in broiler were identified. These QTLs should be considered as novel QTLs may be population-specific.

\section{Positional candidate genes for fat deposition}

We identified 13 PCG in five of the nine QTLs identified (Table 3). In the QTL on GGA5 at $38 \mathrm{Mb}$ we identified the Jun dimerization protein 2 (JDP2) gene. This gene regulates lipid accumulation in adipose tissue acting as a repressor of adipocyte differentiation [63, 64].

We identified PLCG1, HNF4A and FITM2 genes in the QTL on GGA20 at 5-Mb. In human primary adipocytes, Phospholipase C gamma 1 (PLCG1) gene is involved in the calcium signaling pathway. The expression of PLCG1 has been show to affect adipocyte triglyceride content [65]. Hepatocyte nuclear factor-4 $\alpha$ (HNF4A) controls insulin metabolism and triglycerides level [66]. Triglycerides are the main lipid stored in avian fat cells. Thus, different levels of plasma triglyceride may affect fat deposition [67]. The Fat storage inducing transmembrane protein 2 (FITM2/FIT2) gene belongs to a family of proteins that play a role in fat storage [68]. In a study with humans, the FITM2/FIT2 gene was reported to be associated with lipid droplets biogenesis and accumulation [69], which consequently, impacts lipid storage.

In the QTL on GGA26 at $1 \mathrm{Mb}$, we found ADIPOR1, ENSGALG00000000477 and ENSGALG00000000483 genes. In chickens, the adiponectin receptor 1 (ADIPOR1) gene is expressed in fat, liver and muscle, and this gene affect adipocyte differentiation [70]. ADIPOR1 is

Table 4 Characterization of potentially deleterious and high impact SNPs identified in the 13 PCGs

\begin{tabular}{|c|c|c|c|c|}
\hline Associated Gene Name & Ensembl Gene ID & SNP ID & SNP Position $^{a}$ & Consequence \\
\hline FITM2 & ENSGALG00000026285 & rs315805239 & $5,614,711$ & Stop gained \\
\hline Novel gene & ENSGALG00000000483 & rs740555722 & $1,514,268$ & Stop loss \\
\hline PLCG1 & ENSGALG00000003750 & g.5072909A > T & $5,072,909$ & Non-synonymous \\
\hline Novel gene & ENSGALG00000000477 & rs737351616 & $1,509,828$ & Non-synonymous \\
\hline
\end{tabular}

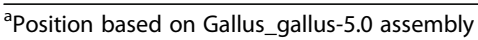


the main receptor of adiponectin. It is negatively correlated with fat deposition [70], and is involved in lipid-induced mitochondrial biogenesis in chicken adipocytes [71]. ENSGALG00000000477 and ENSGALG00000000483 code for uncharacterized proteins, but their gene ontologies are related to lipid metabolism and storage (Table 3). Further studies with those novel genes may help elucidate their role in fat deposition.

In the QTL on GGA15 at $6 \mathrm{Mb}$, we found PTPN11, $M V K$ and ENSGALG00000005043 genes. The protein tyrosine phosphatase, non-receptor 11 (PTPN11) gene encodes for a Src homology-2 domain-containing protein tyrosine phosphatase 2 (SHP2). Its expression has been reported to affect energy balance and lipid and glucose metabolisms [72]. Additionally, in a study with mice, SHP2 was reported to be associated with obesity [73]. Mevalonate kinase $(M V K)$ encodes for a mevalonate kinase enzyme that plays an important role at the beginning of cholesterol biosynthesis [74]. Changes in the cholesterol biosynthesis, and consequently cholesterol levels, may affect hepatic lipid metabolism [75]. ENSGALG00000005043 is a novel gene that has been annotated with GO term related to the fatty acid biosynthetic process and Acetyl-CoA carboxylase activity (Table 3). Further studies with these genes may help to better understand their role in fat deposition.

In the QTL located on GGA24 at $5 \mathrm{Mb}$ we found APOA1, APOA4 and APOA5 genes. These three genes belong to a gene family (Apolipoproteins - APO) that encodes important regulators of lipid biosynthesis and metabolism [76]. Additionally, these three positional genes were annotated with four enriched GO terms: regulation of intestinal cholesterol absorption, high-density lipoprotein particle assembly, lipoprotein metabolic process, and positive regulation of the fatty acid biosynthetic process. Apolipoprotein A1 (APOA1) is involved with cholesterol transport [77]. While, Apolipoprotein A-IV (APOA4) and Apolipoprotein $V(A P O A 5)$ are involved with triglycerides metabolism [76]. Additionally, the APOA4 gene was also reported as a regulator of triglycerides metabolism in mice [77].

Corroborating our findings, no overlap between our positional candidate genes, and genes under selective pressure reported in a previous study with the same dataset [23] was observed.

Additionally, comparing a dataset of SNPs and INDELs identified in Brazilian broiler and layer lines, our group recently identified regions under selection [41], harbouring genes related to fat deposition. No overlap was observed between our PCGs for fat deposition and the genes reported in that study, except for APOA1. Possible explanations for the observed lack of overlap, are the different chicken lines used in these studies, and the removal of fixed SNPs in the current study.

\section{Potential causative SNPs in PCGs}

We observed many SNPs annotated in intronic regions of the PCGs (approximately $42 \%$ of the SNPs; Fig. 3b). According to the literature, introns can play a role in the regulation of alternative splicing, gene expression, and may be associated with mRNA transport [78, 79]. Thus, SNPs annotated in introns can play a role in the regulation of any trait, including fat deposition in chickens.

Approximately 58\% of the SNPs found in the 13 PCG were in potentially functional regions such as up/downstream, 3'and 5'-UTRs, exons (synonymous and non-synonymous), splicing site and stop codon (gained/lost; Fig. 3b). Genetic variants within non-coding regions (3'and 5'-UTRs) may control gene expression by modulating transcription or mRNA turnover [80]. We observed 36 SNPs in 3/5'UTR regions (Fig. 3b). Two of the 35 non-synonymous SNPs were classified as potentially deleterious and were located in PLCG1 and ENSGALG00000000477 genes (Table 4), PCGs for fat deposition regulation. Potentially deleterious SNPs in these genes could be causative mutations.

Two high impact SNPs were annotated in FITM2 and ENSGALG00000000483 genes (Table 4). High impact SNPs in these genes may affect lipid metabolism and storage (fat deposition) in chickens.

From the four SNPs (Table 4), one is novel (g.5072909A > T), and the others are not included on the Affymetrix SNP array. Thus, the integration of GWAS and genome sequencing brought additional information in the search for potential causative mutations. Further studies are necessary to achieve a better understanding of the role of these SNPs in fat deposition.

\section{Conclusions}

This study confirmed previously published QTLs and discovered novel ones, thus contributing to a better understanding of fat deposition in chickens. The use of a high-density SNPs panel in our GWAS analyses provided a better resolution in QTL detection. The PCGs identified in the QTL are involved in many biological processes that regulate lipid storage. We found SNPs located in the PCGs providing additional information in the search for potential causative mutations and further validation studies could be helpful to understand their role in fat deposition regulation. Our findings can be potentially applied to improve the accuracy of early selection against carcass fat accumulation and improve feed efficiency in broiler chicken production.

\section{Additional files}

Additional file 1: Plot of the density of SNPs per Mbp in each autosomal chromosome after filtration. (DOCX $155 \mathrm{~kb}$ ) 
Additional file 2: An excel file with the characterization of all the SNP windows analyzed including the proportion of the genetic variance explained by each one. (ODS $137 \mathrm{~kb}$ )

Additional file 3: Manhattan plot of the posterior means of the proportion of genetic variance explained by each 1-Mb SNP window across the 28 autosomal chromosomes for abdominal fat percentage (ABFP): (A) genomic windows located on macrochromosomes, and (B) windows located on microchromosomes. The $\mathrm{X}$-axis represents the chromosomes and $Y$-axis shows the proportion of genetic variance explained by each window from Bayes B analysis. Red lines indicate the threshold to deem significant SNP windows. (DOCX $245 \mathrm{~kb}$ )

Additional file 4: Manhattan plot of the posterior means of the proportion of genetic variance explained by each 1-Mb SNP window across the 28 autosomal chromosomes for skin weight (SKINW): (A) genomic windows located on macrochromosomes, and (B) windows located on microchromosomes. The X-axis represents the chromosomes and Y-axis shows the proportion of genetic variance explained by each window from Bayes $B$ analysis. Red lines indicate the threshold to deem significant SNP windows. (DOCX $243 \mathrm{~kb}$ )

Additional file 5: Manhattan plot of the posterior means of the proportion of genetic variance explained by each 1-Mb SNP window across the 28 autosomal chromosomes for skin percentage (SKINP): (A) genomic windows located on macrochromosomes, and (B) windows located on microchromosomes. The $\mathrm{X}$-axis represents the chromosomes and $Y$-axis shows the proportion of genetic variance explained by each window from Bayes B analysis. Red lines indicate the threshold to deem significant SNP windows. (DOCX $243 \mathrm{~kb}$ )

Additional file 6: Manhattan plot of the SNP effect distribution within each significant window for abdominal fat percentage (ABFP). The $X$-axis represents the significant SNP window represented by the number of the respective chromosome and Y-axis shows the SNP effect from Bayes B analysis. Their respective start and end positions are: GGA5 $(38,000,437-$ 38,996,916 bp); GGA10 (7,000,336-7,998,549bp); GGA13 (3,002,6173,998,616 bp); GGA26 (1,002,598-1,999,851bp). (DOCX $1425 \mathrm{~kb})$

Additional file 7: Manhattan plot of the SNP effect distribution within each significant window for skin weight (SKINW). The X-axis represents the significant SNP window represented by the number of the respective chromosome and Y-axis shows the SNP effect from Bayes B analysis. Their respective start and end positions are: GGA15 $(6,000,311-6,999,944$ bp); GGA24 (5,000,105-5,999,010 bp); GGA27 (3,000,222-3,997,124bp). (DOCX 1425 kb)

Additional file 8: Manhattan plot of the SNP effect distribution within each significant window for skin percentage (SKINP). The X-axis represents the significant SNP window represented by the number of the respective chromosome and $Y$-axis shows the SNP effect from Bayes B analysis. Their respective start and end positions are: GGA9 $(4,000,836-4,999,336$ bp); GGA15 (6,000,311-6,999,944bp); GGA27 (3,000,222-3,997,124 bp). (DOCX $1425 \mathrm{~kb}$ )

Additional file 9: List with the 419 genes annotated within the nine QTL detected. (XLSX $61 \mathrm{~kb})$

\section{Abbreviations}

ABF: Abdominal Fat Weight; ABFP: Abdominal Fat Percentage; BW42: Body Weight at 42 days of age; DAVID: Database for Annotation, Visualization and Integrated Discovery software; GWAS: Genome-Wide Association Study; HWE: Hardy Weinberg Equilibrium; MAF: Minor Allele Frequency; MCMC: Markov Chain Monte Carlo; NCBI: National Center for Biotechnology Information; OMIM: Online Mendelian Inheritance in Man; PCG: Positional Candidate Gene; PPA: Posterior Probability of Association; QTL: Quantitative Trait Locus; SKINP: Skin Percentage; SKINW: Skin Weight

\section{Acknowledgements}

The authors are grateful to Ricardo Brassaloti (University of Sao Paulo) for the help in genotyping the chickens. The authors are also grateful for the support from the FAPESP, CAPES and CNPq mentioned in funding statement. The authors would like to acknowledge the collaborative efforts among EMBRAPA, University of São Paulo, and lowa State University.

\section{Funding}

This study was supported by the Brazilian Agricultural Research Corporation - Embrapa (project number 01.11.07.002.04.02) and by the thematic project (2014/08704-0) from São Paulo Research Foundation (FAPESP). The TT Reference Population was funded by the National Council of Scientific and Technological Development (CNPq) grant number 481755/2007-1 from the Brazilian Government. Boschiero received a fellowship from the program Science Without Borders - CNPq, grant 370620/2013-5. G.C.M. Moreira received fellowships from FAPESP, grants 14/21380-9 (in cooperation agreement with (APES), and 16/00569-1. T.F. Godoy received fellowship from FAPESP, grant 15/00616-7 (in cooperation agreement with CAPES). L.L. Coutinho is recipient of productivity fellowship from CNPq.

\section{Availability of data and materials}

All data generated or analysed during this study are public and included in this published article. The SNPs reported (identified by sequencing) were submitted to European Variation Archive (EVA) - EMBL-EBI, accession PRJEB25004. The datasets used and/or analysed during the current study (genotypes and phenotypes) are available from the corresponding author on reasonable request.

\section{Authors' contributions}

GCMM, CB, MCL, JMR and LLC conceived the idea of this research and participated in the experimental design. GCMM, CB, TFG, MEC, DG performed data analysis. GCMM drafted the manuscript. GCMM, CB, ASMC, LLC, JMR, DG, TFG, PAT, MEC, MCL, AMGI, ASAMTM and JOP collaborated with interpretation, discussion and writing of the manuscript. AMGl, JOP, MCL, JMR, MEC and LLC provided the experimental environment, phenotype and data analysis support. All authors have read and approved the final manuscript.

\section{Ethics approval}

All experimental protocols related to animal experimentation in this study were performed in agreement with the resolution number 010/2012 approved by the Embrapa Swine and Poultry Ethics Committee on Animal Utilization to ensure compliance with international guidelines for animal welfare.

\section{Competing interests}

Dr. James Reecy is a member of the editorial board (Associate Editor) of BMC Genetics journal.

\section{Publisher's Note}

Springer Nature remains neutral with regard to jurisdictional claims in published maps and institutional affiliations.

\section{Author details}

'Department of Animal Science, University of São Paulo (USP) / Luiz de Queiroz College of Agriculture (ESALQ), Piracicaba, São Paulo 13418-900, Brazil. ${ }^{2}$ Department of Animal Science, lowa State University (ISU), Ames, lowa, USA. ${ }^{3}$ Embrapa Suínos e Aves, Concórdia, Santa Catarina, Brazil. ${ }^{4}$ FMVZ / São Paulo State University, Botucatu, SP, Brazil. ${ }^{5}$ School of Agriculture, Massey University, Ruakura, Hamilton, New Zealand.

Received: 8 December 2017 Accepted: 10 May 2018

Published online: 21 May 2018

\section{References}

1. Resnky CW, Carré W, Wang X, Porter TE, Simon J, Le Bihan-Duval E, et al. Transcriptional analysis of abdominal fat in genetically fat and lean chickens reveals adipokines, lipogenic genes and a link between hemostasis and leanness. BMC Genomics. 2013;14:557.

2. Nassar MK, Goraga ZS, Brockmann G. Quantitative trait loci segregating in crosses between New Hampshire and white leghorn chicken lines: III. Fat deposition and intramuscular fat content. Anim Genet. 2012;1(44):62-8.

3. Zerehdaran S, Vereijken AL, van Arendonk JA, van der Waaijt EH. Estimation of genetic parameters for fat deposition and carcass traits in broilers. Poult Sci. 2004:83:521-5.

4. Ferrini G, Baucells MD, Esteve-García E, Barroeta AC. Dietary polyunsaturated fat reduces skin fat as well as abdominal fat in broiler chickens. Poult Sci. 2008;87(3):528-35. 
5. Jennen DGJ, Vereijken ALJ, Bovenhuis H, Crooijmans RPM, Veenendaal A Van der Poel JJ, et al. Detection and localization of quantitative trait loci affecting fatness in broilers. Poult Sci. 2004;3(83):295-301.

6. Zhou H, Evock-Clover CM, McMurtry JP, Ashwell CM, Lamont SJ. Genome wide linkage analysis to identify chromosomal regions affecting phenotypic traits in the chicken. Poult Sci. 2007:86(2):267-76.

7. Baéza E, Le Bihan-Duval E. Chicken lines divergent for low or high abdominal fat deposition: a relevant model to study the regulation of energy metabolism. Animal: an International Journal of Animal Bioscience. 2013;7:965-73.

8. Wang SZ, Hu XX, Wang ZP, Li XC, Wang QG, Wang YX, et al. Quantitative trait loci associated with body weight and abdominal fat traits on chicken chromosomes 3, 5 and 7. Genet Mol Res. 2012;11:956-65.

9. Ikeobi CO, Woolliams JA, Morrice DR, Law A, Windsor D, Burt DW, et al. Quantitative trait loci affecting fatness in the chicken. Anim Genet. 2002; 33(6):428-35.

10. Lagarrigue S, Pitel F, Carre W, Abasht B, Le Roy P, Neau A, et al. Mapping quantitative trait loci affecting fatness and breast muscle weight in meattype chicken lines divergently selected on abdominal fatness. Genet Sel Evol. 2006;38:85-97.

11. Park H-B, Jacobsson L, Wahlberg P, Siegel PB, Andersson L. QTL analysis of body composition and metabolic traits in an intercross between chicken lines divergently selected for growth. Physiol Genomics. 2006;2(26):216-23.

12. Pinto LFB, Packer IU, Ledur MC, Moura ASAMT. Nones k, Coutinho LL. Mapping quantitative trait loci in Gallus gallus using principal components utilização de componentes principais. Rev Bras Zootec. 2010:2434-41.

13. Nones K, Ledur MC, Zanella EL, Klein C, Pinto LFB, Moura ASAMT, et al. Quantitative trait loci associated with chemical composition of the chicken carcass. Anim Genet. 2012:43:570-6.

14. Rao $Y$, Shen $X$, Xia M, Luo C, Nie Q, Zhang D, et al. SNP mapping of QTL affecting growth and fatness on chicken GGA1. Genetics, selection, evolution: GSE. 2007;39(5):569-82.

15. Abasht B, Dekkers JCM, Lamont SJ. Review of quantitative trait loci identified in the chicken. Poult Sci. 2006;85:2079-96.

16. Sun Y, Zhao G, Liu R, Zheng M, Hu Y, Wu D, et al. The identification of 14 new genes for meat quality traits in chicken using a genome-wide association study. BMC Genomics. 2013;14:458.

17. Liu R, Sun Y, Zhao G, Wang F, Wu D, Zheng M, et al. Genome-wide association study identifies loci and candidate genes for body composition and meat quality traits in Beijing-you chickens. PLoS One. 2013;8(4):e61172.

18. Wang W, Zhang T, Wang J, Zhang G, Wang Y, Zhang Y, et al. Genome-wide association study of 8 carcass traits in Jinghai yellow chickens using specificlocus amplified fragment sequencing technology. Poult Sci. 2016;95(3):500-6.

19. Kranis A, Gheyas AA, Boschiero C, Turner F, Yu L, Smith S, et al. Development of a high density 600K SNP genotyping array for chicken. BMC Genomics. 2013;14:59.

20. Pértille F, Zanella R, Felício AM, Ledur MC, Peixoto JO, Coutinho LL. Identification of polymorphisms associated with production traits on chicken (Gallus gallus) chromosome 4. Genet Mol Res. 2015;14(3):10717-28.

21. Venturini GC, Cruz VA, Rosa JO, Baldi F, El Faro L, Ledur MC, et al. Genetic and phenotypic parameters of carcass and organ traits of broiler chickens. Genet. Mol. Res. 2014:13:10294-300.

22. Fornari MB, Zanella R, Ibelli AMG, Fernandes LT, Cantão ME, Thomaz-Soccol V, et al. Unraveling the associations of osteoprotegerin gene with production traits in a paternal broiler line. Spring. 2014;3:682.

23. Marchesi JAP, Buzanskas ME, Cantão ME, Ibelli AMG, Peixoto JO, Joaquim LB, et al. Relationship of runs of homozygosity with adaptive and production traits in a paternal broiler line. Animal. 2017:1-9.

24. Purcell S, Neale B, Todd-Brown K, Thomas L, Ferreira MA, Bender D, et al. PLINK: a tool set for whole-genome association and population-based linkage analyses. Am J Hum Genet. 2007;81:559-75.

25. Turner S, Armstrong LL, Bradford Y, Carlson CS, Crawford DC, Crenshaw AT, et al. Quality Control Procedures for Genome Wide Association Studies. Curr Protoc Hum Genet. 2011; Chapter 1:Unit1.19.

26. Cesar ASM, Regitano CA, Tullio RR, Lanna DPD, Nassu RT, Mudado MA, et al. Genome-wide association study for intramuscular fat deposition and composition in Nellore cattle. BMC Genet. 2014;15:39.

27. Garrick DJ, Fernando RL. Implementing a QTL detection study (GWAS) using genomic prediction methodology. Methods Molecular Biology. 2013;1019:275-98.

28. Rolf MM, Garrick DJ, Fountain T, Ramey HR, Weaber RL, Decker JE, et al. Comparison of Bayesian models to estimate direct genomic values in multi-breed commercial beef cattle. Genet Sel Evol. 2015;47:23-30.
29. Van Goor A, Bolek KJ, Ashwell CM, Persia ME, Rothschild MF, Schmidt CJ, et al. Identification of quantitative trait loci for body temperature, body weight, breast yield, and digestibility in an advanced intercross line of chickens under heat stress. Genet Sel Evol. 2015:47(1):1-13.

30. Van Goor A, Ashwell CM, Persia ME, Rothschild MF, Schmidt CJ, Lamont SJ. Quantitative trait loci identified for blood chemistry components of an advanced intercross line of chickens under heat stress. BMC Genomics. 2016;17(1):1.

31. Onteru SK, Gorbach DM, Young JM, Garrick DJ, Dekkers JCM, Rothschild MF. Whole genome association studies of residual feed intake and related traits in the pig. PLoS One. 2013;8(6):e61756.

32. Chicken QTL database (release 33), 2017. http://www.animalgenome.org/ cgi-bin/QTLdb/GG/index. Acessed 18 Sept 2017.

33. Flicek P, Ahmed I, Amode MR, Barrell D, Beal K, Brent S, et al. Ensembl 2013 (2013). Nucleic Acids Res. 2013;41(Database issue):D48-55.

34. Kinsella RJ, Kähäri A, Haider S, Zamora J, Proctor G, Spudich G, et al. Ensembl BioMarts: a hub for data retrieval across taxonomic space. Database (Oxford). 2011; https:/doi.org/10.1093/database/bar030.

35. Huang DW, Sherman BT, Lempicki RA. Systematic and integrative analysis of large gene lists using DAVID bioinformatics resources. Nature Protoc. 2009; 4(1):44-57.

36. Huang DW, Sherman BT, Lempicki RA. Bioinformatics enrichment tools: paths toward the comprehensive functional analysis of large gene lists. Nucleic Acids Res. 2009;37(1):1-13.

37. Benjamini $Y$, Hochberg Y. Controlling the false discovery rate: a practical and powerful approach to multiple testing. J R Statist Soc B. 1995;57:289-300.

38. Moreira GCM, Godoy TF, Boschiero C, Gheyas A, Gasparin G, Andrade SCS, et al. Variant discovery in a QTL region on chromosome 3 associated with fatness in chickens. Anim Genet. 2015;46:141-7.

39. Godoy TF, Moreira GCM, Boschiero C, Gheyas AA, Gasparin G, Paduan M, et al. SNP and INDEL detection in a QTL region on chicken chromosome 2 associated with muscle deposition. Anim Genet. 2015;46:158-63.

40. Li H, Handsaker B, Wysoker A, Fennell T, Ruan J, Homer N, et al. The sequence alignment/map format and SAMtools. Bioinformatics. 2009;25(16):2078-9.

41. Boschiero C, Moreira GCM, Gheyas AA, Godoy TF, Gasparin G, Mariani PDSC, et al. Genome-wide characterization of genetic variants and putative regions under selection in meat and egg-type chicken lines. BMC Genomics. 2018; 19(1):83.

42. McLaren W, Pritchard B, Rios D, Chen Y, Flicek P, Cunning-ham F. Deriving the consequences of genomic variants with the Ensembl API and SNP effect predictor. Bioinformatics. 2010;26:2069-70.

43. Predicted data Ensembl - Ensembl (2017). http://www.ensembl.org/info/ genome/variation/predicted_data.html. Accessed 18 Sept 2017.

44. Ng PC, Henikoff S. SIFT: predicting amino acid changes that affect protein function. Nucleic Acids Res. 2003;31:3812-4

45. Abasht B, Pitel F, Lagarrigue S, Le Bihan-Duval E, Le Roy P, Demeure O, et al Fatness QTL on chicken chromosome 5 and interaction with sex. Genet Sel Evol. 2006;38:297-311.

46. Nadaf J, Pitel F, Gilbert H, Duclos MJ, Vignoles F, Beaumont C, et al. QTL for several metabolic traits map to loci controlling growth and body composition in an F2 intercross between high- and low-growth chicken lines. Physiol Genomics. 2009;38(3):241-9.

47. Gao Y, Du ZQ, Wei WH, Yu XJ, Deng XM, Feng CG, et al. Mapping quantitative trait loci regulating chicken body composition traits. Anim Genet. 2009;40(6):952-4.

48. Sheng Z, Pettersson ME, Hu X, Luo C, Qu H, Shu D, et al. Genetic dissection of growth traits in a Chinese indigenous $x$ commercial broiler chicken cross. BMC Genomics. 2013;14:151.

49. Campos RLR, Nones K, Ledur MC, Moura ASAMT, Pinto LFB, Ambo M, et al. Quantitative trait loci associated with fatness in a broiler-layer cross. Anim Genet. 2009:40:729-36.

50. Jennen DG, Vereijken $A L$, Bovenhuis $H$, Crooijmans RM, van der Poel Jر, Groenen MA. Confirmation of quantitative trait loci affecting fatness in chickens. Genetics, Selection, Evolution: GSE. 2005;37(3):215-28.

51. Ankra-Badu GA, Shriner D, Le Bihan-Duval E, Mignon-Grasteau S, Pitel F, Beaumont C, et al. Mapping main, epistatic and sex-specific QTL for body composition in a chicken population divergently selected for low or high growth rate. BMC Genomics. 2010;11:107.

52. de los Campos G, Sorensen D, Gianola D. Genomic Heritability: What Is It? PLoS Genet. 2015;11(5):e1005048.

53. Cahaner A, Nitsan Z. Evaluation of simultaneous selection for live body weight and against abdominal fat in broilers. Poult Sci. 1985;64:1257-63. 
54. Gaya LG, Ferraz JB, Rezende FM, Mourao GB, Mattos EC, Eler JP, Filho TM. Heritability and genetic correlation estimates for performance and carcass and body composition traits in a male broiler line. Poult Sci. 2006;85:837-43.

55. Flisar T, Malovrh S, Tercic D, Holcman A, Kovac M. Thirty-four generations of divergent selection for 8-week body weight in chickens. Poult Sci. 2014;93: 16-23.

56. Wolc A, Arango J, Settar P, Fulton JE, O'Sullivan NP, Preisinger R, Habier D, Fernando R, Garrick DJ, Dekkers JCM. Persistence of accuracy of genomic estimated breeding values over generations in layer chickens. Genet Sel Evol. 2011;43:23-10.

57. Wolc A, Stricker C, Arango J, Settar P, Fulton JE, O'Sullivan NP, Preisinger R, Habier D, Fernando R, Garrick DJ. Breeding value prediction for production traits in layer chickens using pedigree or genomic relationships in a reduced animal model. Genet Sel Evol. 2011;43:5-10.

58. Wolc A, Arango J, Jankowski T, Dunn I, Settar P, Fulton JE, et al. Genomewide association study for egg production and quality in layer chickens. J Anim Breed Genet. 2014;131(3):173-82.

59. Wolc A, Arango J, Jankowski T, Settar P, Fulton JE, O'Sullivan NP, et al. Genome-wide association study for Marek's disease mortality in layer chickens. Avian Dis. 2013;57:395-400.

60. Fernando RL, Garrick DJ. Bayesian methods applied to GWAS. Methods Molecular Biology. 2013;1019:237-74.

61. Sheng ZY, Pettersson ME, Honaker CF, Siegel PB, Carlborg O. Standing genetic variation as a major contributor to adaptation in the Virginia chicken lines selection experiment. Genome Biol. 2015;16:219.

62. Mackay TF. Q\&A: Genetic analysis of quantitative traits. J Biol. 2009;8(3):23.

63. Nakade K, Pan J, Yoshiki A, Ugai H, Kimura M, Liu B, et al. JDP2 suppresses adipocyte differentiation by regulating histone acetylation. Cell death Diff. 2007;14:1398-405.

64. Huang Y-C, Hasegawa H, Wang S-W, Ku C-C, Lin Y-C, Chiou S-S, et al. Jun dimerization protein 2 controls senescence and differentiation via regulating histone modification. J Biomed Biotechnol. 2011;2011:569034

65. Shen W, Martinez K, Chuang CC, Mclntosh M. The phospholipase C inhibitor U73122 attenuates trans-10, cis-12 conjugated linoleic acid-mediated inflammatory signaling and insulin resistance in human adipocytes. J Nutr. 2013;143(5):584-90.

66. Krapivner S, Iglesias MJ, Silveira A, Tegnér J, Björkegren J, Hamsten A, et al. DGAT1 participates in the effect of HNF4A on hepatic secretion of triglyceriderich lipoproteins. Arterioscler Thromb Vasc Biol. 2010;30(5):962-7.

67. Griffin H. Understanding genetic variation in fatness in chickens. Annual Report 95/96 Roslin Institute 1996;35-38.

68. Kadereit B, Kumar P, Wang W-J, Miranda D, Snapp EL, Severina N, et al. Evolutionarily conserved gene family important for fat storage. Proc Natl Acad Sci U S A. 2008;105(1):94-9.

69. Choudhary V, Ojha N, Golden A, Prinz WA. A conserved family of proteins facilitates nascent lipid droplet budding from the ER. J Cell Biol. 2015;211(2): 261-71.

70. Yan J, Yang H, Gan L, Sun C. Adiponectin-impaired adipocyte differentiation negatively regulates fat deposition in chicken. J Anim Physiol Anim Nutr (Berl). 2014;98:530-7

71. Gan L, Yan J, Liu Z, Feng M, Sun C. Adiponectin prevents reduction of lipidinduced mitochondrial biogenesis via AMPKJACC2 pathway in chicken adipocyte. J Cell Biochem. 2015;116:1090-100

72. Nagata N, Matsuo K, Bettaieb A, Bakke J, Matsuo I, Graham J, et al. Hepatic Src homology phosphatase 2 regulates energy balance in mice. Endocrinology. 2012;153(7):3158-69.

73. Krajewska M, Banares S, Zhang EE, Huang X, Scadeng M, Jhala US, et al. Development of Diabesity in mice with neuronal deletion of Shp2 tyrosine phosphatase. Am J Pathol. 2008:172(5):1312-24.

74. Willer CJ, Sanna S, Jackson AU, Scuteri A, Bonnycastle LL, Clarke R, et al. Newly identified loci that influence lipid concentrations and risk of coronary artery disease. Nat Genet. 2008;40(2):161-9.

75. Flock MR, Green MH, Kris-Etherton PM. Effects of adiposity on plasma lipid response to reductions in dietary saturated fatty acids and cholesterol. Adv Nutr. 2011;2(3):261-74.

76. Delgado-Lista J, Perez-Jimenez F, Ruano J, Perez-Martinez P, Fuentes F, Criado-Garcia J, et al. Effects of variations in the APOA1/C3/A4/A5 gene cluster on different parameters of postprandial lipid metabolism in healthy young men. J Lipid Res. 2010;51(1):63-73.
77. Baroukh N, Bauge E, Akiyama J, Chang J, Afzal V, Fruchart JC, et al. Analysis of apolipoprotein A5, C3, and plasma triglyceride concentrations in genetically engineered mice. Arterioscler Thromb Vasc Biol. 2004;24:1297-302.

78. Jo BS, Choi SS. Introns: the functional benefits of introns in genomes. Genomics Inform. 2015;13(4):112-8.

79. Berulava T, Horsthemke B. The obesity-associated SNPs in intron 1 of the FTO gene affect primary transcript levels. Eur J Hum Genet. 2010;18:1054-6.

80. Chatterjee S, Pal JK. Role of 50 - and 30 -untranslated regions of mRNAs in human diseases. Biol Cell. 2009;101:251-62.

\section{Ready to submit your research? Choose BMC and benefit from:}

- fast, convenient online submission

- thorough peer review by experienced researchers in your field

- rapid publication on acceptance

- support for research data, including large and complex data types

- gold Open Access which fosters wider collaboration and increased citations

- maximum visibility for your research: over $100 \mathrm{M}$ website views per year

At BMC, research is always in progress.

Learn more biomedcentral.com/submissions 